CLINICAL STUDY

\title{
Amiodarone-induced thyrotoxicosis: left ventricular dysfunction is associated with increased mortality
}

Anthony J O'Sullivan, Mridula Lewis and Terrance Diamond

Departments of Medicine and Endocrinology, St George Hospital and University of New South Wales, Kogarah, Sydney, New South Wales 2217, Australia

(Correspondence should be addressed to A J O'Sullivan; Email: A.OSullivan@unsw.edu.au)

\begin{abstract}
Objective: Amiodarone-induced thyrotoxicosis (AIT) is a challenging management problem, since patients treated with amiodarone invariably have underlying heart disease. Consequently, thyrotoxicosis can significantly contribute to increased morbidity and mortality. The aim of this study was to compare the clinical outcome and hormone profiles of patients with AIT $(n=60)$ with those with Graves' thyrotoxicosis $(n=49)$ and toxic multinodular goitre (MNG, $n=40)$.

Design: A retrospective study of patients with AIT in a single institution was conducted.

Methods: Data from patients with AIT over 12 years were collected.

Results: Mean TSH levels were significantly suppressed in all three groups. However, there was no intergroup significant difference. Free thyroxine (T4) levels were significantly higher in AIT $(45.6 \pm 3.5 \mathrm{pmol} / \mathrm{l})$ and Graves' disease $(44.6 \pm 4.0 \mathrm{pmol} / \mathrm{l})$ compared with toxic MNG (31.5 $\pm 5.1 \mathrm{pmol} / \mathrm{l}, P<0.05)$. In contrast, free triiodothyronine (T3) levels were only significantly higher in Graves' disease $(14.7 \pm 1.5 \mathrm{pmol} / \mathrm{l}, P=0.002)$ compared with AIT $(8.6 \pm 0.7 \mathrm{pmol} / \mathrm{l})$ and toxic MNG (7.4 $\pm 0.5 \mathrm{pmol} / \mathrm{l})$. Six deaths occurred in the patients with AIT $(10.0 \%, P<0.01)$ and no deaths occurred in the other groups. Amiodarone treatment $(P=0.002)$ was the most significant predictor of death, whereas free T4, free T3 and age did not affect outcome. Within the amiodarone-treated group severe left ventricular dysfunction $(P=0.0001)$ was significantly associated with death.

Conclusions: (i) AIT differs from other forms of thyrotoxicosis, and (ii) severe left ventricular dysfunction is associated with increased mortality in AIT.
\end{abstract}

European Journal of Endocrinology 154 533-536

\section{Introduction}

Amiodarone, an iodine-rich ( $37 \%$ by weight) benzofuran derivative, has been in use for nearly 20 years $(1,2)$. The main therapeutic role for amiodarone is in the management of cardiac arrhythmias, both atrial and ventricular $(3,4)$, where amiodarone has been shown to reduce the risk of sudden death or cardiac arrest (4). Hence, most patients who require treatment with amiodarone have underlying cardiac dysfunction, but not all have reduced ventricular function (5). Amiodarone-induced thyroid dysfunction has been reported to affect between 2 and $24 \%$ of users, but most investigators believe amiodarone affects less than $5 \%$ of users $(1-3)$. Amiodarone results in thyroid dysfunction due to intrinsic properties of amiodarone as well as iodine-induced effects (2). The risk of thyroid dysfunction is reported to be increased with exposure to higher cumulative doses of amiodarone (6). Amiodarone-induced thyrotoxicosis (AIT) remains a challenging problem with significant morbidity, due to worsening arrthymias (3) and even mortality (7). Medical management can be problematic, prompting the use of different medical treatments $(5,6,8)$, plasmapheresis $(9,10)$ and surgery in some cases $(11,12)$, especially when drug-unresponsive $(13,14)$. However, the factors which are associated with mortality from AIT are less well defined.

The aim of the study was to compare the clinical outcome and hormonal profiles of patients with different forms of thyrotoxicosis due to amiodarone, Graves' disease and toxic multinodular goitre (MNG).

\section{Patients and methods}

A retrospective analysis was performed in a single Institution, St George Hospital. All patients from 1992 to 2004 who were diagnosed with AIT were included in the study. The diagnosis of AIT $(n=60)$ was based on the following criteria: significant previous amiodarone 
use (greater than 4 weeks) still continuing at diagnosis, biochemical hyperthyroidism including a raised free thyroxine (T4) level and a suppressed thyrotrophin (TSH) level, clinical signs of hyperthyroidism, reduced thyroid radioisotope uptake and a negative anti-TSH receptor antibody $(2,3,15)$. AIT was not differentiated into Type I and II $(15,16,17)$. The treating physicians used anti-thyroid medication and corticosteroids according to their clinical discretion $(15,16,18)$. Of the 60 patients with AIT, nine were treated with anti-thyroid medication (carbimazole 20-40 mg daily) plus corticosteroids (prednisone $30-50 \mathrm{mg} /$ day) alone. Thyroid surgery was required in 11 patients, three of whom required plasmapheresis, who responded poorly to medical management and the remainder were treated with carbimazole 20-40 mg daily only. Patients with Graves' disease $(n=49)$ were defined on the following criteria: biochemical hyperthyroidism, clinical signs of hyperthyroidism including a uniform smooth goitre, a uniform thyroid radioisotope uptake and positive thyroid receptor antibodies (19). Patients with Graves' disease were all treated with anti-thyroid medication (carbimazole $10-40 \mathrm{mg}$ daily), 15 also received radioactive iodine and four were surgically treated. Patients with toxic MNG $(n=40)$ were defined on the following criteria: biochemical hyperthyroidism, clinical signs of hyperthyroidism including an irregular goitre and heterogeneous thyroid radioisotope uptake (19). The patients with toxic MNG were all treated with carbimazole $5-30 \mathrm{mg}$ daily and 27 received radioactive iodine.

A cross-sectional comparison of all three groups was made. Patients with Graves' thyrotoxicosis and toxic MNG who fulfilled the diagnostic criteria were selected and all patients were de-identified. The demographics of all three groups are shown in Table 1. An outcome of death was recorded if the patient died before the normalization of the free T4 levels. Cumulative amiodarone dose was calculated as the total dose of amiodarone up to the time of diagnosis of thyrotoxicosis as amiodarone treatment ceased that this time.

All subjects with AIT underwent transcutaneous echocardiography within 6 weeks of their diagnosis of thyrotoxicosis as part of their assessment for their cardiac disease. Left ventricular function was classified as normal if the ejection fraction was greater than $55 \%$, mild dysfunction if left ventricular function was $45-55 \%$, moderate dysfunction if it was $30-45 \%$ and severe dysfunction if it was less than 30\% (20). TSH was measured by a third-generation, solid-phase, two-site chemiluminescent immunometric assay (BioMediq DPC Immulite 2000; Diagnostics Products Corporation, Los Angeles, CA, USA) with a coefficient of variation $(\mathrm{CV})$ of $3.7 \%$. Free T4 was measured by a competitive analog immunoassay with a CV of $4.1 \%$. Free triiodothyronine (T3) was measured by a competitive analog-based immunoassay with a $\mathrm{CV}$ of $7.0 \%$. Statistical analysis was performed with the aid of Statview using an ANOVA model. Relationships between variables were determined by multiple regression analysis. Results are expressed as means \pm S.E.M.

\section{Results}

Patients with Graves' disease were significantly younger than the patients with AIT and toxic MNG (see Table 1). Graves' thyrotoxicosis and toxic MNG predominantly occurred in women compared with AIT, where men were preferentially affected.

Mean TSH levels were significantly suppressed in all three groups. However, there were no significant intergroup differences (see Table 2). Free T4 levels were significantly higher in AIT (45.6 $3.5 \mathrm{pmol} / \mathrm{l})$ and Graves' disease $(44.6 \pm 4.0 \mathrm{pmol} / \mathrm{l})$ compared with toxic $\mathrm{MNG}$ $(31.5 \pm 5.1 \mathrm{pmol} / \mathrm{l}, \quad P<0.05)$. In contrast, free T3 levels were only significantly higher in Graves' disease $(14.7 \pm 1.5 \mathrm{pmol} / \mathrm{l}, \quad P=0.002)$ compared with AIT $(8.6 \pm 0.7 \mathrm{pmol} / \mathrm{l})$ and toxic $\mathrm{MNG}(7.4 \pm 0.5 \mathrm{pmol} / \mathrm{l})$. Thus, the free T4 to free T3 ratio was increased in AIT (7.3 $\pm 0.5, P=0.001)$ compared with Graves' disease (3.5 \pm 0.2$)$ and toxic MNG $(4.1 \pm 0.4)$, reflecting the reduced conversion of T4 to T3 which is seen in amiodarone-induced thyroid dysfunction $(1,2)$. The cumulative amiodarone dose ranged from 6 to $584 \mathrm{~g}$ (mean $122.7 \pm 13.8 \mathrm{~g}$ ). The time to normalize free T4 levels was significantly longer in the AIT group (27.9 \pm 3.5 weeks, $P<0.01$ ) compared with Graves' thyrotoxicosis (12.7 \pm 1.4 weeks) and toxic MNG (8.0 \pm 0.5 weeks), reflecting the relative resistance to medical therapy seen with AIT (see Table 1).

Table 1 Demographic characteristics, cumulative amiodarone dosage, time to normalize free T4 levels and outcome in patients with amiodarone-induced thyrotoxicosis (AIT), Graves' thyrotoxicosis (Graves') and toxic multinodular goitre (TMNG). Means \pm S.E.M.

\begin{tabular}{lccc}
\hline & AIT & Graves' & TMNG \\
\hline Number of patients & 60 & 49 & 40 \\
Age (years) & $68.3 \pm 1.7$ & $42.8 \pm 2.3^{\#}$ & $72.7 \pm 2.1$ \\
Sex (male/female) & $42 / 18 \S$ & $11 / 38$ & $1 / 39$ \\
Cumulative amiodarone dose (g) & $122.7 \pm 13.8^{\S}$ & 0 & 0 \\
Duration of amiodarone therapy (days) & $409 \pm 46^{\S}$ & 0 & 0 \\
Time to normalize free T4 (weeks) & $27.9 \pm 3.5^{\natural}$ & $12.7 \pm 1.4$ & 0 \\
Outcome (deaths) & $6^{\pi}$ & $0.0 \pm 0.5$ \\
\hline
\end{tabular}

${ }^{\#} P=0.001$ Graves' vs AIT and TMNG; ${ }^{\S} P=0.001$ AIT vs Graves' and TMNG; ${ }^{~} P<0.01$ AIT vs Graves' and TMNG. 
Table 2 Thyroid hormone levels in patients with amiodarone-induced thyrotoxicosis (AIT), Graves' thyrotoxicosis (Graves') and toxic multinodular goitre (TMNG). Means \pm S.E.M.

\begin{tabular}{lccc}
\hline & AIT & Graves' & TMNG \\
\hline TSH $(\mathrm{mU} / \mathrm{l})$ & $0.06 \pm 0.02$ & $0.01 \pm 0.01$ & $0.07 \pm 0.03$ \\
Free T4 (pmol/l) & $45.6 \pm 3.5$ & $44.6 \pm 4.0$ & $31.5 \pm 5.1^{\#}$ \\
Free T3 (pmol/l) & $8.6 \pm 0.7$ & $14.7 \pm 1.5^{\S}$ & $7.4 \pm 0.5$ \\
Free T4/T3 ratio & $7.3 \pm 0.5$ & $3.5 \pm 0.2$ & $4.1 \pm 0.4$ \\
Anti-thyroid peroxidase antibody $(\%+\mathrm{ve})$ & 7 & 32 & 13 \\
\hline
\end{tabular}

${ }^{\#} P<0.05$ TMNG vs Graves' and AIT; ${ }^{\S} P=0.002$ Graves' vs AIT and TMNG; ${ }^{\natural} P=0.0001$ AIT vs Graves' and TMNG.

Six deaths occurred in the patients with AIT $(10.0 \%$, $P<0.01)$ before free T4 levels were normalized. Four deaths occurred due to intractable left ventricular dysfunction and two due to multi-organ failure, one in a patient with end-stage renal failure and another with significant pulmonary fibrosis. No deaths occurred in the other two groups. AIT was a significant predictor of death $(P=0.002)$, whereas free T4, free T3 and age were not. Within the group with AIT, severe left ventricular dysfunction $(P=0.0001)$ and age $(P=0.005)$ were significant predictors of death, whereas sex, free T4, free T3 and cumulative amiodarone dose were not. Of the 12 patients with AIT and severe left ventricular dysfunction, six died $(50 \%)$. No patients with mild or moderate left ventricular dysfunction or normal left ventricular function died.

\section{Discussion}

This study demonstrates that AIT is more common in men compared with other forms of thyrotoxicosis, which are more common in women $(19,21,22)$, probably reflecting a higher incidence of cardiac disease in men (2). AIT was also associated with a higher free T4 to free T3 ratio, supporting studies which show that amiodarone reduces the peripheral conversion of $\mathrm{T} 4$ to T3 due to amiodarone inhibiting type I iodo-thyronine 5'-deiodinase (1). AIT was more resistant to medical therapy, consistent with a more prolonged time to normalize free T4 levels, possibly due to the high intrathyroidal iodine content, which reduces the effectiveness of thionamide therapy (1). In this study, patients with AIT were at increased risk of death, especially if they were elderly with severe left ventricular dysfunction. In contrast, the severity of the thyrotoxicosis did not appear to increase mortality in AIT. The increased mortality in the patients with severe left ventricular dysfunction is most probably related to their advanced cardiac disease rather than the drug amiodarone.

In the present study, AIT was associated with significant mortality, with $10 \%$ of the group dying before their thyrotoxicosis was under control, compared with no deaths in the two other groups with thyrotoxicosis. Wong et al. (7) reviewed 24 cases of AIT where five patients died, resulting in a $20.8 \%$ mortality. Since patients on amiodarone have underlying cardiac dysfunction (14) and often other co-morbidities, it is no surprise mortality is high when thyrotoxicosis, a pro-arrhythmic condition, develops (23). Moreover, the sensitivity of the cardiovascular system to develop arrthymias and cardiac failure in thyrotoxicosis is increased in the presence of pre-morbid cardiac dysfunction (23). In our subgroup of 12 patients with severe left ventricular dysfunction, six died (50\% mortality). Once again, this is not surprising as a left ventricular ejection fraction of less than $40 \%$ is a powerful predictor of mortality in patients with ischaemic heart disease (20). In AIT, age was also associated with increased mortality, probably related to increased incidence of other co-morbidities in the ageing population.

AIT has been divided into excess iodine-induced thyroid hormone synthesis in an abnormal gland (Type I) and destructive thyroiditis (Type II) (15-17), but more recently a group with features of Type I and Type II (mixed) has been recognized (2). Type I is associated with ongoing thyrotoxicosis and therefore treatment with thionamides is required, whereas Type II is treated with corticosteroids (15). The difficulty clearly differentiating Type I and II has led authors to suggest therapy should be directed towards both types until the diagnosis seems certain $(2,24)$. It is unclear whether the mortality is different between the two types of AIT. Treatment of AIT is not straightforward. Because of the high iodine content of amiodarone and the low radioiodine uptake on scanning, radioiodine ablative therapy is ineffective compared with other forms of thyrotoxicosis (24). Bartalena et al. (16) successfully treated 12 patients with Type I AIT with methimazole $30 \mathrm{mg} /$ day plus perchlorate, observing a normalization of T3 levels by 4 weeks. In contrast, their 12 patients with Type II AIT all responded to $40 \mathrm{mg} /$ day prednisone. In another study, patients with Type II AIT were randomized to iopanoic acid $1 \mathrm{~g}$ daily or prednisone $30 \mathrm{mg}$ daily (15). Although, both medications were effective is rapidly reducing T3 levels, free T4 levels did not normalize for 221 days for iopanoic acid and 43 days for prednisone. In contrast, 28 cases of AIT were reviewed by Osman et al. (25), where five cases spontaneously resolved and 23 responded to carbimazole treatment, although 
adjunctive steroids were introduced into one patient who was resistant to thioamide alone. Surgery has been advocated as effective management with one study reporting successful treatment of AIT with thyroidectomy, where the authors report surgery as lifesaving in the three most critical cases (13). Other groups have reported similar responses to surgery, especially when unresponsive to medical therapy $(11,14)$.

The present study was retrospective and prospective studies are required to confirm the findings. Moreover, the patients with AIT were not stratified into Type I or II and more data are required to determine whether mortality is different between the two Types. Treatment was not randomized and therefore it was not possible to conclude whether one treatment modality was better than another.

AIT remains a challenging management condition. The findings in the present study support the use of an echocardiogram in assessing the patient. If severe left ventricular dysfunction is present, aggressive medical treatment should ensue, and surgery may be considered early. Patients with moderate-severe left ventricular dysfunction may also require monitoring of the thyroid function more regularly. We conclude that (i) AIT differs from other forms of thyrotoxicosis, and (ii) severe left ventricular dysfunction is associated with increased mortality in patients with AIT.

\section{Acknowledgements}

We would like to thank the Endocrinology Laboratory, South Eastern Area Laboratory Services for doing the hormonal assays and the Department of Cardiology, St George Hospital for doing the echocardiography.

\section{References}

1 Harjai KJ \& Licata AA. Effects of amiodarone on thyroid function. Annals of Internal Medicine 1997126 63-67.

2 Basaria S \& Cooper DS. Amiodarone and the thyroid. American Journal of Medicine 2005118 706-714.

3 Martino E, Bartalena L, Bogazzi F \& Braverman LE. The effects of amiodarone on the thyroid. Endocrine Reviews 200122 240-254.

4 Campbell TJ. The place of amiodarone: an overview of the four recent large controlled trials. Australian and New Zealand Journal of Medicine $199727582-590$.

5 Roy D, Talajic M, Dorian P, Connelly S, Eisenberg MJ, Green M, Kus T, Lambert J, Dubuc M, Gagne P, Nattel S \& Thibault T. Amiodarone to prevent recurrence of atrial fibrillation. New England Journal of Medicine $2000342913-920$.

6 Bouvy ML, Heerdink ER, Hoes AW \& Leufkens HG. Amiodaroneinduced thyroid dysfunction associated with cumulative dose. Pharmacoepidemiology and Drug Safety 200211 601-602.

7 Wong R, Cheung W, Stockigt JR \& Topliss DJ. Heterogeneity of amiodarone-induced thyrotoxicosis: evaluation of colour-flow Doppler sonography in predicating therapeutic response. Internal Medicine Journal 200333 420-426.
8 Newnham HH, Topliss DJ, Le Grand BA, Chosich N, Harper RW \& Stockigt JR. Amiodarone-induced hyperthyroidism: assessment of the predictive value of biochemical testing and response to combined therapy using propylthiouracil and potassium perchlorate. Australian and New Zealand Journal of Medicine 198818 37-43.

9 Diamond TH, Rajagopal R, Ganda K, Manoharan A \& Luk A. Plasmapheresis as a potential treatment option for amiodaroneinduced thyrotoxicosis. Internal Medicine Journal $2004 \mathbf{3 4}$ $369-370$.

10 Ligtenberg J, Tulleken J \& Zijlstra J. Plasmapheresis in thyrotoxicosis. Annals of Internal Medicine 1999131 71-72.

11 Gough IR \& Gough J. Surgical management of amiodaroneassociated thyrotoxicosis. Medical Journal of Australia $2002 \mathbf{1 7 6}$ $128-129$.

12 Bogazzi F, Miccoli P, Berti P, Cosci C, Brogioni S, AghiniLombardi F, Materazzi G, Bartalena L, Pinchera A, Braverman LE \& Martino E. Preparation with iopanoic acid rapidly controls thyrotoxicosis in patients with amiodarone-induced thyrotoxicosis before thyroidectomy. Surgery 2002132 1114-1117.

13 Hamoir E, Meurisse M, Defechereux T, Joris J, Vivario J \& Hennen G. Surgical management of amiodarone-associated thyrotoxicosis: too risky or too effective? World Journal of Surgery 199822 537-543.

14 Claxton S, Sinha SN, Donovan S, Greenaway TM, Hoffman L, Loughhead M \& Burgess JR. Refractory amiodarone associated thyrotoxicosis: an indication for thyroidectomy. Australian and New Zealand Journal of Surgery 200070 174-178.

15 Bogazzi F, Bartalena L, Cosci C, Brogioni S, Dell'Unto E, Grasso L, Aghini-Lombardi F, Rossi G, Pinchera A, Braverman LE \& Martino E. Treatment of Type II Amiodarone-induced thyrotoxicosis by either iopanoic acid or glucocorticoids: a prospective, randomized study. Journal of Clinical Endocrinology and Metabolism 199988 1999-2002.

16 Bartalena L, Brogioni S, Grasso L, Bogazzi, Burelli A \& Martino E. Treatment of amiodarone-induced thyrotoxicosis, a difficult challenge: results of a prospective study. Journal of Clinical Endocrinology and Metabolism 199681 2930-2933.

17 Bartalena L, Grasso L, Brogioni S, Aghini-Lombardi F, Braverman LE \& Martino E. Serum interleukin-6 in amiodarone-induced thyrotoxicosis. Journal of Clinical Endocrinology and Metabolism $1994 \mathbf{7 8} 423-427$.

18 Reichert LJ \& de Rooy HA. Treatment of amiodarone induced hyperthyroidism with potassium perchlorate and methimazole during amiodarone treatment. British Medical Journal 1989298 1547-1548.

19 Lazarus JH. Hyperthyroidism. Lancet 1997349 339-343.

20 Schiller NB \& Foster E. Analysis of left ventricular function. British Heart Journal 199675 17-26.

21 Albert SG, Alves LE \& Rose EP. Thyroid dysfunction during chronic amiodarone therapy. Journal of the American College of Cardiology 19879 175-183.

22 Harjai KJ \& Licata AA. Amiodarone-induced hyperthyroidism: a case series and brief review of literature. Pacing and Clinical Electrophysiology $1996191548-1554$.

23 Ladeson PW. Thyrotoxicosis and the heart: something old and something new. Journal of Clinical Endocrinology and Metabolism $199377332-333$.

24 Daniels GH. Amiodarone-induced thyrotoxicosis. Journal of Clinical Endocrinology and Metabolism 200186 3-8.

25 Osman F, Franklyn JA, Sheppard MC \& Gammage MD. Successful treatment of amiodarone-induced thyrotoxicosis. Circulation 2002 $1051275-1277$.

Received 14 November 2005

Accepted 13 January 2006 